\title{
Conocimientos, conductas y toma de decisiones de médicos pediatras sobre hipoacusia infantil en tres hospitales del Servicio de Salud Viña del Mar-Quillota
}

\author{
Understanding of paediatricians' knowledge, behaviours \\ and decision-making on paediatric hearing loss in three hospitals \\ from the Viña del Mar-Quillota Health Service
}

Felipe Retamal-Walter ${ }^{1,2}$, Andre Gómez-Lombardi³, Gonzalo Martínez-Zelaya ${ }^{4}$

\section{Resumen}

Introducción: Entre todas las dificultades sensoriales, la hipoacusia es la más prevalente a nivel mundial. Algunas de sus consecuencias incluyen dificultades de lenguaje, aprendizaje, conducta, peor desempeño académico y baja autoestima. La participación de médicos pediatras es clave en el seguimiento del desarrollo auditivo, lingüístico y comunicativo infantil. Objetivo: Conocer y describir el conocimiento sobre hipoacusia de pediatras del Servicio de Salud Viña del Mar-Quillota, Chile. Materiales y Método: Investigación transversal-descriptiva. Treinta y tres médicos de los servicios de Neonatología y Pediatría del Servicio de Salud Viña del Mar-Quillota completaron un cuestionario de evaluación de conocimientos generales y manejo de hipoacusia. Resultados: Los participantes reconocieron la importancia de su rol con respecto al seguimiento comunicativo infantil. Sin embargo, el $72 \%$ de los participantes reportó recibir formación sobre audiología e hipoacusia únicamente durante su formación de pregrado. Más del $50 \%$ de los participantes identificó correctamente todos los factores de riesgo de hipoacusia, demostrando así sus conocimientos sobre detección temprana. Se reportó la necesidad de mayores conocimientos sobre tipos y clasificaciones de hipoacusia, pruebas de evaluación y marco legal de la hipoacusia en Chile. Conclusión: La entrega de mayor información sobre hipoacusia a médicos pediatras, junto con oportunidades de formación continua al cursar la especialidad de pediatría y durante el ejercicio profesional es fundamental para la identificación y seguimiento adecuados de la hipoacusia. Estas medidas favorecerían la detección e intervención temprana de hipoacusia y reducirían potencialmente el impacto y las consecuencias en el desarrollo de niños y niñas con hipoacusia.

Palabras clave: hipoacusia infantil; detección temprana de hipoacusia; conocimiento; pediatras; audiología.

\footnotetext{
Abstract

Introduction: Of all diagnosable sensory impairments, hearing loss $(H L)$ has the highest prevalence worldwide. Consequences of HL include but are not limited to communication disability, language and learning disorders, behavioural difficulties, academic underachievement, and low self-esteem. Therefore, paediatrician involvement in ongoing care of paediatric HL is crucial for auditory, linguistic, and communicative aspects of childhood development. Aim: To understand and describe the knowledge about paediatric HL of paediatricians from the Viña del Mar-Quillota Health Service, Chile. Material and Method: A descriptive cross-sectional study was employed. Thirty-three doctors from Neonatology and Paediatric Units from the Viña del Mar-Quillota Health Service completed a questionnaire exploring knowledge and behaviours regarding paediatric HL. Results: Participants acknowledged the importance of their role in the continuous care of paediatric HL. However, $72 \%$ of participants were provided with knowledge on paediatric HL only throughout their
}

\author{
${ }^{1}$ Escuela de Ciencias de \\ la Salud y Rehabilitación, \\ Universidad de Queensland, \\ Australia. \\ ${ }^{2}$ Hospital Dr. Gustavo Fricke. \\ Viña del Mar, Chile. \\ ${ }^{3}$ Escuela de Fonoaudiología, \\ Universidad de Valparaíso. \\ Valparaíso, Chile. \\ ${ }^{4}$ Escuela de Ciencias Jurídicas \\ y Sociales, Universidad Viña \\ del Mar. Viña del Mar, Chile. \\ Los autores declaran no tener \\ conflictos de interés. \\ Recibido el 24 de enero de \\ 2020. Aceptado el 4 de julio \\ de 2020. \\ Correspondencia: \\ Felipe Retamal-Walter \\ 84A Services Rd, St Lucia, QLD \\ 4067. Australia. \\ Email: f.retamalwalter@ \\ uq.net.au
}


post-secondary education. Over $50 \%$ of participants identified all HL risk factors, demonstrating the acquisition of foundational knowledge of early HL detection. Gaps in knowledge identified by participants included types and classification of HL, types of assessments, and policies and procedures to guide treatment of paediatric HL in Chile. Conclusion: Provision of HL information to paediatricians, and ongoing professional development is vital to the decisions regarding ongoing care of paediatric HL. Potential outcomes of knowledge provision and ongoing professional development for paediatric residents and paediatricians may result in early $H L$ detection and intervention of paediatric $H L$, potentially reducing the impact of associated developmental consequences for children with HL.

Keywords: Paediatric hearing loss; early hearing detection; knowledge; paediatricians; audiology.

\section{Introducción}

La hipoacusia es descrita como la dificultad sensorial más prevalente a nivel mundial ${ }^{1}$, con aproximadamente 34 millones de niños y niñas experimentando alguna hipoacusia incapacitante alrededor del mundo ${ }^{2}$. La incidencia de la hipoacusia congénita es variable y ha sido estimada entre 1 y 3 casos por cada 1.000 nacidos vivos ${ }^{3-5}$. Algunas de las consecuencias reportadas en casos de niños y niñas con hipoacusia permanente incluyen dificultades de lenguaje, habla, aprendizaje y problemas conductuales $^{6}$, peor desempeño académico ${ }^{7}$ y baja autoestima ${ }^{8,9}$.

Una de las problemáticas más relevantes de la salud pública actual es la detección precoz de la hipoacusia infantil y, para ello, se sugiere la ejecución de programas de tamizaje auditivo universal ${ }^{10,11}$. Aquellos programas de tamizaje auditivo selectivo para poblaciones con factores de riesgos son útiles, sin embargo, insuficientes. Alrededor de la mitad de las hipoacusias congénitas presentan antecedentes de riesgo conocidos por el cual ser estudiadas, sin embargo, la otra mitad no los posee ${ }^{12} \mathrm{y}$, por tanto, podrían ser subdiagnosticadas. En regiones sin programas de tamizaje auditivo universal la edad promedio de detección de la hipoacusia es entre los 2,5 y 3 años ${ }^{13-15}$.

El rol de los pediatras es clave dentro del equipo multidisciplinario para detectar una hipoacusia tempranamente. Los pediatras son quienes deben permanecer vigilantes ante señales tempanas de hipoacusia ${ }^{16}$, tanto en atención primaria (AP) como secundaria y terciaria. Tanto en países que cuentan con tamizaje auditivo universal, como para aquellos niños y niñas diagnosticados 'normoyentes' luego del primer examen auditivo, son los pediatras quienes continúan el seguimiento del desarrollo por varios años. Por lo tanto, su liderazgo y compromiso facilitarían la vigilancia permanente de la hipoacusia, particularmente para casos de hipoacusia tardía o progresiva ${ }^{16}$.

En Chile, el sistema sanitario ha sido definido como hospitalocéntrico, con una tendencia hacia la promoción, desarrollo y formación de especialistas a nivel terciario, dejando a la AP con un bajo número de especialistas ${ }^{17}$. El mismo sistema ha sido descrito como un sistema fragmentado ${ }^{17}$, asegurando el diagnóstico y tratamiento de enfermedades de alta morbilidad a través de su programa Garantías Explícitas en Salud (GES) ${ }^{18}$. Dichas GES dependen administrativamente de los Servicios de Salud (servicios descentralizados representantes regionales del Ministerio de Salud). El programa GES, al día de hoy, no contempla el tamizaje auditivo universal y describe dos programas selectivos para la identificación de la hipoacusia: 1) detección y tratamiento de la hipoacusia en recién nacidos prematuros; y 2) tratamiento de la hipoacusia moderada, severa y profunda en personas menores de 4 años ${ }^{18,19}$. En Chile, la AP es administrada a nivel municipal y no depende, por tanto, del Sistema Nacional de Servicios de Salud. Debido al tipo de financiamiento, la mercantilización y fragmentación del sistema de salud, sumado a la escasez de especialistas en AP y a la ausencia de tamizaje auditivo universal, se dificulta el diagnóstico oportuno y la vigilancia auditiva rutinaria de niños y niñas durante sus controles de salud.

\section{Objetivo}

Debido a la relevancia de los pediatras con respecto al desarrollo motor, lingüístico y 
comunicativo infantil y la exposición de pediatras de la atención terciaria con la legislación chilena vigente sobre hipoacusia ${ }^{19}$, el objetivo de esta investigación fue conocer y describir el conocimiento sobre hipoacusia infantil de los médicos pediatras funcionarios del Servicio de Salud Viña del Mar-Quillota, Chile.

\section{Material y Método}

Investigación de carácter transversal-descriptivo. La población de médicos participantes fue identificada y estuvo conformada por el total de médicos funcionarios de la Red Asistencial del Servicio de Salud Viña del Mar-Quillota cuyos hospitales contasen con servicios clínicos de neonatología y pediatría durante 2017-2018. Debido a lo anterior, los participantes de esta investigación debían cumplir los siguientes criterios de inclusión: médico pediatra, subespecialista pediátrico o residente cursando especialidad en pediatría con al menos 1 año de antigüedad; y rol de pediatra tratante o interconsultor en el servicio clínico de neonatología y/o pediatría de los hospitales participantes.

La recolección de los datos se llevó a cabo mediante la aplicación de un cuestionario de evaluación formado por preguntas cerradas y abiertas, con la opción de complementar la respuesta en los casos correspondientes. El cuestionario se compuso de dos partes: la primera consistió en la recopilación demográfica y formación académica de los participantes y la segunda en un cuestionario de conocimientos generales sobre hipoacusia, procedimientos de evaluación audiológica, toma de decisiones y marco legal de la hipoacusia en Chile. En total 48 cuestionarios fueron entregados en 3 hospitales de la red asistencial, de los cuales 33 cuestionarios fueron contestados por los participantes.

Dicho cuestionario ha sido utilizado previamente en otras investigaciones cuyo objetivo fue evaluar el conocimiento de pediatras sobre hipoacusia ${ }^{20-22}$. Debido que el cuestionario original fue desarrollado en portugués ${ }^{20}$, este fue traducido y adaptado al español chileno para ser utilizado en esta investigación. La validez de contenido ${ }^{23}$ fue realizada por la Unidad de Otorrinolaringología del Hospital Dr. Gusta- vo Fricke y por un panel de fonoaudiólogos expertos en audiología de la Universidad de Valparaíso. Antes de aplicar el cuestionario se realizó un pilotaje $\mathrm{e}^{24}$, con el objetivo de recibir retroalimentación sobre la estructura, redacción y claridad de las preguntas y el diseño del instrumento.

Esta investigación contó con la aprobación de Comité de Ética acreditado (resolución 15/2017). Luego de obtener aprobación ética, el investigador principal se acercó a los jefes de los servicios de neonatología y pediatría de tres hospitales del Servicio de Salud Viña del Mar-Quillota para discutir la divulgación del cuestionario entre potenciales participantes. Todos los participantes debían consentir por escrito su participación voluntaria en esta investigación. Se utilizó una pauta de corrección del cuestionario desarrollada por el equipo de investigación en conjunto con la Unidad de Otorrinolaringología del Hospital Dr. Gustavo Fricke. Los resultados del instrumento se analizaron a través de estadística descriptiva, principalmente a través de frecuencias absolutas y frecuencias relativas porcentuales. Se utilizaron los programas de análisis estadístico STATA 15 y Microsoft Excel Professional 2016.

\section{Resultados}

\section{Información demográfica y formación académica de los participantes}

Treinta y tres participantes $(68,75 \%)$ completaron satisfactoriamente el cuestionario de evaluación, de un total de 48 instrumentos entregados. En la Tabla 1 se detalla la información demográfica y la formación profesional y académica en audiología de los participantes.

\section{Conocimientos generales de audiología infantil, evaluación audiológica, toma de decisiones y marco legal de la hipoacusia en Chile}

En relación con factores de riesgo de hipoacusia, los participantes debían identificar aquellos factores de riesgo conocidos de hipoacusia en población infantil. En la Tabla 2 se reportan aquellos factores de riesgo identificados por los participantes, ordenados de manera decreciente. 


\section{ARTÍCULO DE INVESTIGACIÓN}

\section{Tabla 1. Información demográfica de los participantes}

\begin{tabular}{|c|c|c|}
\hline & $\mathbf{n}$ & $\%$ \\
\hline \multicolumn{3}{|l|}{ Especialidad médica de los participantes $(n=33$ ) } \\
\hline Pediatría & 27 & 81,81 \\
\hline \multicolumn{3}{|l|}{ Subespecialidad pediátrica } \\
\hline Broncopulmonar & 1 & 3,03 \\
\hline Cardiología & 1 & 3,03 \\
\hline Neurología & 1 & 3,03 \\
\hline Puericultura & 1 & 3,03 \\
\hline No declara especialidad & 2 & 6,06 \\
\hline \multicolumn{3}{|l|}{ Número de años de experiencia profesional desde titulación $(n=30)$} \\
\hline $1-5$ años & 3 & 10,00 \\
\hline $6-10$ años & 7 & 23,33 \\
\hline $11-15$ años & 2 & 6,67 \\
\hline 16 - 20 años & 5 & 16,67 \\
\hline $21-25$ años & 2 & 6,67 \\
\hline Más de 25 años & 11 & 36,67 \\
\hline \multicolumn{3}{|l|}{ Lugar de trabajo principal $(n=33)$} \\
\hline Hospital público & 30 & 90,91 \\
\hline Clínica privada & 3 & 9,09 \\
\hline Atención primaria & 0 & 0,00 \\
\hline Consulta particular & 0 & 0,00 \\
\hline \multicolumn{3}{|l|}{ Servicio o unidad de desempeño profesional $(n=26)$} \\
\hline Unidad de Paciente Crítico/Cuidado Intensivo Neonatal o Pediátrico & 10 & 38,46 \\
\hline Unidad de Emergencia Infantil & 5 & 19,23 \\
\hline Unidad de Recién Nacido Intermedio & 1 & 3,85 \\
\hline Unidad de Pediatría / Servicio de Pediatría / Pediatría atención abierta & 6 & 23,08 \\
\hline \multicolumn{3}{|l|}{ Otro } \\
\hline Segunda infancia & 1 & 3,85 \\
\hline Policlínico de especialidades & 1 & 3,85 \\
\hline Hematooncología & 1 & 3,85 \\
\hline Servicio quirúrgico pediatría & 1 & 3,85 \\
\hline \multicolumn{3}{|l|}{ Formación académica en audiología ( $n=33$ ) } \\
\hline Únicamente clases de pregrado sobre audición e hipoacusia & 24 & 72,72 \\
\hline Clases de postgrado/postítulo sobre audición e hipoacusia & 4 & 12,12 \\
\hline \multicolumn{3}{|l|}{ Formación mixta sobre audición e hipoacusia } \\
\hline Clases de pregrado y cursos específicos & 1 & 3,03 \\
\hline Clases de pregrado, postgrado y asistencia a congresos & 2 & 6,06 \\
\hline \multicolumn{3}{|l|}{ Módulos específicos sobre audición (especifique) } \\
\hline Curso presencial en hospital de referencia otorrino de la región (10 años atrás) & 1 & 3,03 \\
\hline Curso online tratamiento de la pérdida auditiva en el paciente pediátrico & 1 & 3,03 \\
\hline
\end{tabular}




\begin{tabular}{|c|c|c|}
\hline Factor de riesgo de hipoacusia & $\mathbf{n}$ & $\%$ \\
\hline Infección por meningitis bacteriana/encefalitis viral ${ }^{*}$ & 33 & 100 \\
\hline Síndromes asociados con hipoacusia* & 32 & 96,97 \\
\hline Antibióticos ototóxicos por más de 5 días* & 32 & 96,97 \\
\hline Historia familiar de hipoacusia* & 30 & 90,91 \\
\hline Antibióticos ototóxicos por más de 5 días (aminoglucósidos)* & 30 & 90,91 \\
\hline Infección connatal - rubéola* & 28 & 84,85 \\
\hline Encefalopatía hipóxico isquémica perinatal aguda ${ }^{\dagger}$ & 28 & 84,85 \\
\hline Peso de nacimiento $<1.500 \mathrm{~g}^{\dagger}$ & 27 & 81,88 \\
\hline Malformaciones craneofaciales* & 27 & 81,82 \\
\hline Enfermedades neurodegenerativas ${ }^{*}$ & 26 & 78,79 \\
\hline Stress neonatal ${ }^{+}$ & 25 & 75,77 \\
\hline Traumatismo de base de cráneo o fractura temporal ${ }^{*}$ & 25 & 75,76 \\
\hline Estadía en UCIN por más de 5 días* $^{*}$ & 24 & 72,73 \\
\hline Terapia con oxigenación de membrana extracorpórea* & 22 & 66,67 \\
\hline Quimioterapia* & 22 & 66,67 \\
\hline Infección connatal - citomegalovirus* & 21 & 63,64 \\
\hline Hiperbilirrubinemia que requiera exanguinotransfusión* & 21 & 63,64 \\
\hline Septicemia neonatal severa* & 20 & 60,61 \\
\hline Infección connatal - toxoplasmosis* & 18 & 54,55 \\
\hline Infección connatal - herpes* & 18 & 54,55 \\
\hline Ventilación mecánica por más de 5 días* & 18 & 54,55 \\
\hline Infección connatal - sífilis* & 16 & 48,48 \\
\hline Hiperbilirrubinemia* & 11 & 33,33 \\
\hline Estadía en UCI por más de $48 \mathrm{~h}^{\dagger}$ & 3 & 9,09 \\
\hline
\end{tabular}

Con respecto a las preguntas relacionadas con la evaluación audiológica, el 84,85\% de los participantes mencionaron solicitar una evaluación o seguimiento audiológico específico en niños y niñas con alto riesgo de hipoacusia. Adicionalmente, un 42,42\% reportó evaluar rutinariamente la audición de sus pacientes durante atenciones ambulatorias. De ellos, el $39,39 \%$ reportó utilizar pruebas objetivas para determinar el estado auditivo de sus pacientes, mencionando el uso de potenciales evocados auditivos de tronco cerebral, potenciales evocados automáticos y emisiones otoacústicas. El
51,52\% de los participantes reportaron utilizar pruebas subjetivas para evaluar la audición de sus pacientes. Algunos ejemplos de las pruebas subjetivas mencionadas fueron: respuesta al sonido de una campana; seguimiento de la voz con la mirada; respuesta ante ruidos fuertes, chasquido de los dedos y el tic-tac de un reloj.

Frente a la pregunta sobre la conducta de los pediatras ante la inquietud de los padres sobre el estado auditivo de su hijo(a), el 69,70\% mencionó realizar una evaluación subjetiva de la audición durante la misma atención y complementar dicha evaluación con otra prueba 
(ej., examen auditivo objetivo; derivación a otorrinolaringología y/o fonoaudiología). Ante la pregunta: ¿cuándo deriva a un niño(a) a una evaluación de la audición?, el 90,91\% de los participantes reportó hacerlo ante presencia de factores de riesgo de hipoacusia, mientras que el 84,85\% reportó derivar a evaluación de la audición al quedar con sospechas durante la examinación física. Un $69,70 \%$ reportó hacerlo cuando el padre o madre presentaban inquietudes sobre la audición de su hijo(a), mientras que el 6,06\% de los participantes reportó derivar a evaluación auditiva como procedimiento de rutina.

En la Tabla 3 se presentan las respuestas de los participantes ante las preguntas: ¿a partir de qué edad solicita la primera evaluación formal de la audición a sus pacientes? y ¿cuál piensa usted es la edad más adecuada para derivar a un niño(a) a un especialista de la audición en presencia de factores de riesgo de hipoacusia.

Las respuestas de los participantes ante la pregunta: ¿a partir de cuál edad usted piensa

\section{Tabla 3. Edad habitual de solicitud evaluación audiológica versus edad apropiada de solicitud evaluación audiológica en presencia de factores de riesgo de hipoacusia infantil}

n $\%$

¿A partir de qué edad usted solicita la primera evaluación formal de la audición en sus pacientes? $(n=28)$

$$
\begin{aligned}
& 0-3 \text { meses } \\
& 3-6 \text { meses } \\
& 6-12 \text { meses } \\
& 12-24 \text { meses }
\end{aligned}
$$

Después de los 2 años

Después de los 3 años

¿Cuál edad piensa usted es la más adecuada para derivar a un niño(a) a un especialista de la audición cuando existen factores de riesgo de hipoacusia? $(n=32)$

\begin{tabular}{lrr}
$0-3$ meses & 26 & 81,25 \\
$3-6$ meses & 4 & 12,50 \\
$6-12$ meses & 1 & 3,13 \\
$12-24$ meses & 1 & 3,13 \\
Después de los 2 años & 0 & 0,00 \\
Después de los 3 años & 0 & 0,00 \\
\hline
\end{tabular}

que un niño(a) puede usar ayudas auditivas?, reportaron que el 59,38\% de ellos respondió entre 0-6 meses de edad. Un 18,75\% reportó oportuno comenzar a usar ayudar auditivas entre los 6-12 meses de edad y otro 18,75\% entre los 12-24 meses de edad. Un 3,13\% de los participantes reportó los 2 años de edad como punto de inicio para usar ayudas auditivas.

El cuestionario de evaluación incluyó dos preguntas generales sobre clasificación de la hipoacusia. Un $27,27 \%$ de los participantes afirmaron conocer alguna clasificación de la hipoacusia de acuerdo con su severidad (ej., hipoacusia leve, moderada, severa y profunda ${ }^{25}$ ), sin embargo, el 3,03\% detalló correctamente dicha clasificación. Respecto de la clasificación de hipoacusia por tipo (es decir, daño oído medio - hipoacusia de conducción; daño oído interno - hipoacusia neurosensorial; daño oído medio e interno - hipoacusia mixta ${ }^{26}$ ), el 78,79\% afirmó conocer dichos tipos de hipoacusia. De ellos, un 26,92\% detalló correctamente dicha clasificación, mientras que un $73,08 \%$ lo hizo de manera incompleta. Los participantes expresaron su grado de acuerdo o desacuerdo ante la afirmación "es labor del médico neonatólogo/pediatra preocuparse sobre los aspectos comunicativos de los infantes y niños". Las respuestas de los participantes fueron expresadas en una escala de 1 a 5 y se detallan en la Figura 1.

Los participantes fueron preguntados sobre conductas a seguir en cuatro escenarios de niños y niñas con hipoacusia. Los escenarios incluían 3 tipos de hipoacusia presentadas de manera unilateral o bilateral sin especificación del grado de la patología. Cada escenario era de respuesta abierta, por lo que los participantes podían justificar sus respuestas. La mayoría de los participantes respondió derivar a otorrinolaringólogo en al menos el 81,82\% de los casos para los cuatro escenarios presentados. Al ser una pregunta de respuesta abierta, algunos participantes detallaron conductas adicionales. De 48 respuestas complementarias entre los diferentes escenarios, se describieron 21 interconsultas a neurología infantil $(43,75 \%)$, 17 interconsultas a fonoaudiología $(35,42 \%)$, 4 derivaciones a escuelas de lenguaje $(8,33 \%)$, 2 candidaturas a implante coclear $(4,17 \%)$ y 1 otoscopía $(2,08 \%)$. 

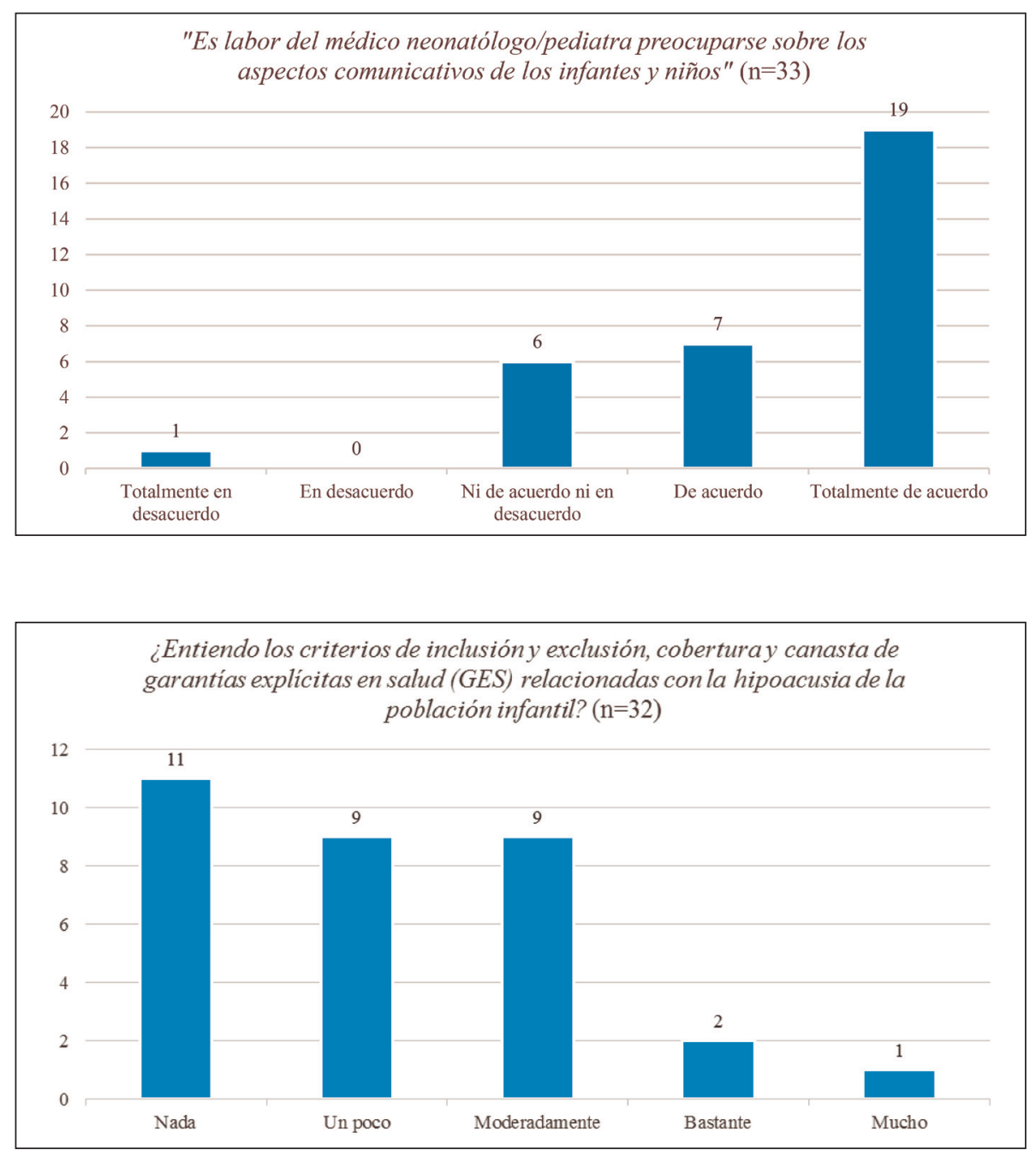

Figura 1. Autopercepción del rol del médico neonatólogo o pediatra en relación al desarrollo comunicativo de los niños.
Figura 2. Nivel de comprensión de médicos neonatólogos, pediatras o residentes de pediatría sobre las Garantías Explícitas en Salud (GES) relacionadas con hipoacusia infantil.
Frente a la pregunta: ¿conozco las GES relacionadas con hipoacusia infantil?, un $30,30 \%$ reportó conocerlas y un $54,55 \%$ desconocerlas. De aquellos participantes que declararon conocer dichas garantías, el 50\% nombró correctamente ambas garantías de hipoacusia y el 40\% nombró únicamente la garantía de hipoacusia del prematuro. Finalmente, los participantes debían expresar su grado de desacuerdo o acuerdo ante la pregunta ¿entiendo los criterios de inclusión y exclusión, cobertura y canasta de las GES relacionadas con la hipoacusia de la población infantil? Las respuestas de los participantes fueron expresadas en una escala de 1 a 5 y se detallan en la Figura 2.

\section{Discusión}

El objetivo de esta investigación fue conocer y describir el conocimiento sobre hipoacusia infantil de médicos pediatras de los servicios de neonatología y pediatría de tres hospitales pertenecientes al Servicio de Salud Viña del Mar-Quillota. Los resultados demostraron un conocimiento actualizado de los pediatras en relación con la identificación de factores de riesgo específico de hipoacusia, aun cuando la mayoría de los participantes reportó recibir instrucción sobre audiología e hipoacusia únicamente durante sus estudios de pregrado. Estos resultados son similares a los reportados por la literatura respecto 
a la falta de entrenamiento en audiología e hipoacusia durante la educación médica ${ }^{27,28}$. Si bien los resultados demostraron que los pediatras reconocieron la mayoría de los factores de riesgo asociados con la aparición de hipoacusia durante la primera infancia, algunos factores de riesgo identificados por los participantes han sido descartados como causantes de hipoacusia ${ }^{29}$.

Al medir el conocimiento sobre la clasificación de las hipoacusias por tipo y grado de severidad, los participantes reportaron un conocimiento parcial. Sólo el 26,92\% de los participantes describió correctamente la clasificación de hipoacusia por tipo y el 3,03\% describió correctamente la clasificación por grado. Estos resultados son similares a los reportados por la literatura ${ }^{22,27,28}$, con profesionales de la salud reconociendo la necesidad de expandir sus conocimientos sobre hipoacusia y las consecuencias de esta. Algunas de las medidas que incrementarían la instrucción y/o concientización de médicos pediatras sobre audiología infantil incluyen: 1) la inclusión dentro del currículo de la especialidad de pediatría de aquellos contenidos relacionados con audiología, lenguaje y comunicación infantil; 2) la ejecución de pasantías por policlínicos de otorrinolaringología y fonoaudiología durante el curso de la especialidad de pediatría; 3 ) la participación de pediatras tanto durante el curso de su especialización en pediatría como durante el ejercicio de su profesión en cursos de perfeccionamiento presenciales $\mathrm{u}$ online en audiología infantil facilitados por profesionales especialistas (ej., médicos otorrinolaringólogos, fonoaudiólogos, tecnólogos médicos en otorrinolaringología); 4) la ejecución de programas colaborativos entre universidades que imparten la especialidad de pediatría y aquellos campos clínicos en AP de salud, de manera que aquellos médicos cursando especialidad en pediatría desarrollen como parte de sus habilidades clínicas la evaluación rutinaria de la audición y comunicación infantil y 5) el establecimiento de colaboraciones entre sociedades médicas y/o colegios profesionales interesados (ej., Sociedad Chilena de Pediatría, Sociedad Chilena de Otorrinolaringología, Colegio Chileno de Fonoaudiólogos) para favorecer instancias de educación interprofesional que faciliten la actualización en el conocimiento sobre audiología infantil de los médicos pediatras.

La mayoría de los participantes $(84,85 \%)$ mencionaron solicitar una evaluación específica en presencia de factores de riesgo de hipoacusia, sin embargo, sólo un 6,06\% reportó derivar a evaluación auditiva a todos sus pacientes como procedimiento de rutina. A pesar de la correcta derivación reportada por los pediatras ante factores de riesgo de hipoacusia, la evidencia ha demostrado que aproximadamente el $50 \%$ de hipoacusias no presenta factores de riesgo asociados ${ }^{12}$.

La mayoría de los participantes $(78,79 \%)$ señaló estar de acuerdo o completamente de acuerdo con la afirmación "es labor del pediatra preocuparse sobre los aspectos comunicativos de infantes y niños". El rol del pediatra ha sido descrito como esencial dentro del equipo multidisciplinario ${ }^{16,28,30}$, siendo responsable de las derivaciones iniciales a evaluación auditiva y el posterior seguimiento del desarrollo infantil ${ }^{16,31}$. Se sugiere por tanto que, ante la ausencia de tamizaje auditivo universal, los pediatras adopten la derivación a evaluación auditiva como un procedimiento de rutina.

Sorpresivamente, el 51,52\% de los participantes mencionó utilizar pruebas subjetivas para evaluar la audición de sus pacientes y sólo el 39,39\% reportó solicitar pruebas objetivas fisiológicas (potenciales evocados auditivos y/o emisiones otoacústicas) como herramientas de tamizaje auditivo. Estos hallazgos reportan la falta de estandarización en los mecanismos preventivos de detección de hipoacusia en población infantil y han sido previamente reportados ${ }^{22}$. La evidencia es clara con respecto al momento y tipo de evaluación sugerida como tamizaje auditivo. Todos los recién nacidos deben acceder a un examen auditivo dentro del primer mes de vida mediante pruebas fisiológicas ${ }^{11,30}$. Adicionalmente, e independiente del resultado del examen inicial, todos los niños y niñas con señales de retraso en el desarrollo de sus habilidades auditivas y/o comunicativas o ante la preocupación de los padres deben recibir una evaluación audiológica formal para descartar la presencia de hipoacusia ${ }^{30}$. El uso de pruebas subjetivas como herramienta 
de evaluación auditiva presenta numerosas limitaciones que disminuyen su validez (ej., dependencia de edad y estado del niño(a); variabilidad interexaminador; dificultad para detectar hipoacusia leve y moderada) y está únicamente recomendada de manera complementaria junto con pruebas fisiológicas a partir de los 6 meses de edad ${ }^{32}$.

Más de la mitad de los participantes reportaron los primeros 6 meses de vida como la edad en que niños y niñas con hipoacusia pueden utilizar ayudas auditivas. Las recomendaciones establecidas en el Position Statement del Joint Committee on Infant Hearing sugieren que todos los infantes con hipoacusia reciban servicios de intervención temprana (ayudas auditivas -audífonos/implante coclear- y rehabilitación auditiva) antes de los 6 meses de edad ${ }^{30}$. Los resultados de la investigación demuestran la conciencia existente entre la mayoría de los pediatras sobre la detección e intervención temprana de la hipoacusia. Resultados similares han sido reportados en la literatura sobre la concientización, conductas $\mathrm{y}$ actitudes de profesionales de la salud sobre programas de tamizaje auditivo universal, diagnóstico e intervención temprana de la hipoacusia ${ }^{21,22,27,28,33}$.

Con respecto al conocimiento del marco legal de la hipoacusia en Chile, llama la atención que el 30,30\% de los participantes reportaron conocer las GES relacionadas con hipoacusia y sólo el 15,15\% las nombró correctamente. Un 54,55\% de los participantes respondió desconocer los programas de detección de hipoacusia y el $62,50 \%$ reportó entender "nada" o "un poco" sobre los criterios de inclusión, exclusión, cobertura y prestaciones incluidas en la legislación vigente (Figura 2). Tanto el desconocimiento de los profesionales de la salud como la poca claridad en los mecanismos regulatorios, legislación y prácticas habituales en la detección e intervención de la hipoacusia han sido reportados previamente $e^{16,21,22,27,28}$. Urge, por tanto, direccionar los requerimientos expresados por los pediatras sobre su contribución en audiología pediátrica. Esto permitiría mejorar sus conocimientos y preparación ante casos de hipoacusia, colaborando en el proceso de detección temprana, intervención oportuna y seguimiento según las recomendaciones vigentes. Adicionalmente, y ante la eventual incorporación de un programa de tamizaje auditivo universal en Chile, es de vital importancia preparar a todos los participantes involucrados en el desarrollo infantil, destacando el rol de los pediatras en el seguimiento comunicativo, fundamentalmente, durante el control del niño sano provisto en AP, atenciones a nivel secundario, terciario y dentro del sistema de salud privado.

Los autores reconocen la existencia de ciertas limitaciones durante el desarrollo de esta investigación. Los resultados solo representan a la población estudiada, sin poder extrapolar los resultados a otros servicios de salud del país o en países con programas de tamizaje auditivo universal. Adicionalmente, los servicios de neonatología y pediatría investigados presentan distintos niveles de complejidad y recursos, por tanto, las respuestas de los participantes pueden haber sido afectadas por las características propias de cada establecimiento.

En futuras investigaciones, se recomienda evaluar a nivel nacional el conocimiento y razonamiento clínico en la toma de decisiones sobre hipoacusia de médicos generales $y$ pediatras encargados del control sano y seguimiento de niños y niñas en AP. Esto permitiría diseñar estrategias de educación e intervención transversales para disminuir las brechas existentes en la formación de especialistas en pediatría, favoreciendo la detección e intervención temprana de aquellos niños y niñas con hipoacusia.

\section{Agradecimientos}

A todos los participantes, por su tiempo y honestidad en sus respuestas, cuyo único afán es mejorar la calidad de la atención y el cuidado integral de nuestros niños y niñas. Tanto al equipo de otorrinolaringología del Hospital Dr. Gustavo Fricke como a los profesionales del Departamento de Audiología de la Escuela de Fonoaudiología de la Universidad de Valparaíso, por su retroalimentación en el desarrollo y validación del cuestionario de evaluación. A quienes colaboraron con la distribución y recolección de las encuestas y, especialmente, a la Fonoaudióloga Paula Beláustegui por su colaboración en la tabulación de los datos. 


\section{Bibliografía}

1. Newton V. Paediatric Audiological Medicine. Hoboken, United Kingdom: John Wiley \& Sons, Incorporated; 2009.

2. World Health Organization. Global estimates on prevalence of hearing loss. Geneva 2018; Available from: http://www.who.int/deafness/Global-estimateson-prevalence-of-hearing-loss-for-website.pptx?ua=1

3. McLeod S, Press F, Phelan C. The (In)visibility of Children with Communication Impairment in Australian Health, Education, and Disability Legislation and Policies. Asia Pacific Journal of Speech, Language and Hearing. 2010;13(1):67-75.

4. Mehra S, Eavey R, Keamy D. The epidemiology of hearing impairment in the United States: Newborns, children, and adolescents. Los Angeles, CA: SAGE Publications; 2009. p. 461-72.

5. Ching T, Oong R, van Wanrooy E. The Ages of Intervention in Regions with and without Universal Newborn Hearing Screening and Prevalence of Childhood Hearing Impairment in Australia. Australian and New Zealand Journal of Audiology. 2006;28(2):137-50.

6. Choi S, Kei J, Wilson W. Rates of hearing loss in primary school children in Australia: A systematic review. Speech, Language and Hearing. 2017;20(3):154-62.

7. McCormack J, Harrison L, McLeod S, McAllister L. A nationally representative study of the association between communication impairment at 4-5 years and children's life activities at 7-9 years. J Speech Lang Hear Res. 2011;54(5):1328-48.

8. Roland L, Fischer C, Tran K, Rachakonda T, Kallogjeri D, Lieu J. Quality of life in children with hearing impairment: systematic review and metaanalysis. Otolaryngology-Head and Neck Surgery. 2016;155(2):208-19.

9. Nordvik $\varnothing$, Heggdal P, Brännström J, Vassbotn F, Aarstad A, Aarstad H. Generic quality of life in persons with hearing loss: a systematic literature review. BMC Ear, Nose and Throat Disorders. 2018;18(1):1.

10. Nelson H, Bougatsos C, Nygren P. Universal newborn hearing screening: systematic review to update the 2001 US Preventive Services Task Force Recommendation. Pediatrics. 2008;122(1):e266-e76.

11. Sedano C, San Martín U, Rahal M. Realidad nacional de los programas de detección auditiva temprana con miras a la cobertura universal. Rev Otorrinolaringol Cir Cabeza Cuello. 2018;78(1):9-14.

12. Olusanya B, Newton V. Global burden of childhood hearing impairment and disease control priorities for developing countries. The Lancet. 2007;369(9569):1314-7.

13. Shulman S, Besculides M, Saltzman A, Ireys H, White K, Forsman I. Evaluation of the universal newborn hearing screening and intervention program. Pediatrics. 2010;126(Supplement 1):S19-S27.

14. Grill E, Hessel F, Siebert U, Schnell-Inderst P, Kunze S, Nickisch A, et al. Comparing the clinical effectiveness of different new-born hearing screening strategies. A decision analysis. BMC Public Health. 2005;5(1):12.

15. Wolff R, Hommerich J, Riemsma R, Antes G, Lange $\mathrm{S}$, Kleijnen J. Hearing screening in newborns: systematic review of accuracy, effectiveness, and effects of interventions after screening. Arch Dis Child. 2010;95(2):130-5.

16. Olusanya B. Neonatal hearing screening and intervention in resource-limited settings: an overview. Arch Dis Child. 2012;97(7):654-9.

17. Centro de Políticas Públicas UC. Fortalecimiento de la atención primaria de salud: propuestas para mejorar el sistema sanitario chileno. Centro de Políticas Públicas UC, 2014 Contract No.: 67.

18. Ley N ${ }^{\circ} 19966$. Establece un Régimen de Garantías en Salud. Diario Oficial de la República de Chile, Santiago, Chile, 25 de agosto de 2004. p. 1-15.

19. Decreto $N^{\circ} 42449$. Aprueba Garantías Explícitas en Salud del Régimen General de Garantías en Salud. Diario Oficial de la República de Chile, Santiago, Chile, 1 de julio de 2019. p. 1-93.

20. Barros A, Galindo M, Jacob R. Conhecimento e conduta de pediatras frente à deficiência auditiva. Pediatria (Säo Paulo). 2002;24(1/2):25-31.

21. Colozza P, Anastasio A. Avaliação, diagnóstico e tratamento da surdez-conhecimento e conduta de médicos com atuação em neonatologia e/ou pediatria de um hospital-escola terciário. Sao Paulo Med J. 2009; 127:61-5.

22. Campos A, Shirane H, Takemoto P, Lourenço E. Universal newborn hearing screening: knowledge of pediatricians and neonatologists in the city of Jundiaí, São Paulo, Brazil. Braz J Otorhinolaryngol. 2014;80(5):379-85.

23. Heale R, Twycross A. Validity and reliability in quantitative studies. Evid Based Nurs. 2015;18(3):667.

24. Leung W-C. How to design a questionnaire. Student BMJ. 2001:187.

25. World Health Organization. Grades of hearing impairment. n.d.; Available from: https://www.who. int/deafness/hearing_impairment_grades/en/

26. Ministerio de Salud de Chile. Guía Clínica Auge: Hipoacusia Neurosensorial Bilateral del Prematuro. Santiago: MINSAL; 2010 [cited 201921 Oct]; Available from: https://www.minsal.cl/portal/url/ item/721fc45c97379016e04001011f0113bf.pdf

27. Ravi R, Gunjawate D, Yerraguntla K, Rajashekhar B. Systematic review of knowledge of, attitudes towards, and practices for newborn hearing screening among healthcare professionals. Int J Pediatr Otorhinolaryngol. 2018;104:138-44. 
ARTÍCULO DE INVESTIGACIÓN

28. Moeller M, White K, Shisler L. Primary care physicians' knowledge, attitudes, and practices related to newborn hearing screening (medical practice). Pediatrics. 2006;118(4):1357.

29. Núñez-Batalla F, Trinidad-Ramos G, Sequí-Canet JM, Alzina De Aguilar V, Jáudenes-Casaubón C. Risk factors for sensorineural hearing loss in children. Acta Otorrinolaringol Esp. 2012;63(5):382390. doi:10.1016/j.otorri.2011.02.007

30. Joint Committee on Infant Hearing. Year 2019 Position Statement: Principles and Guidelines for Early Hearing Detection and Intervention Programs. Journal of Early Hearing Detection and Intervention. 2019;4(2):1-44.

31. Ministerio de Salud Gobierno de Chile. Norma técnica para la supervisión de niños y niñas de
0 a 9 años en la Atención Primaria de Salud 2014 octubre 2019. Disponible en: https:// www.minsal.cl/sites/default/files/files/2014_ Norma\%20T\%c3\%a9cnica\%20para $\% 201$ a $\% 20$ supervisi\%c3\%b3n\%20de\%20ni\%c3\%blos\%20y\%20 ni\%c3\%blas\%20de\%200\%20a\%209\%20en\%20 APS_web(1).pdf

32. Widen JE, Keener SK. Diagnostic testing for hearing loss in infants and young children. Ment Retard Dev Disabil Res Rev. 2003;9(4):220-224. doi:10.1002/ mrdd. 10083

33. Johnson C, Danhauer J, Granali A, Ross M, Harrison J, Cresawn C. Systematic Review of Physicians' Knowledge of, Participation in, and Attitudes toward Newborn Hearing Screening Programs. Seminars In Hearing. 2009;30(3):149-64. 